\title{
The influence of power electronic converter passive filter parameters for flicker in LED lighting
}

\author{
Dariusz Sobczynski ${ }^{1, *}$ \\ ${ }^{1}$ Rzeszow University of Technology, Department of Power Electronics and Power Engineering, The \\ Faculty of Electrical and Computer Engineering, 35-959 Rzeszow, Poland
}

\begin{abstract}
The light emitting diodes (LEDs) are ever more used, and their price is getting lower year on year. Although, exchanging traditional light sources for the LED lamps does not seem to be complicated, we need to remember to maintaining high-quality of light. One of the aspects we should be worry about is a light's flickering. Due to the shape of the current-voltage characteristics of the LED diode and their dependence on temperature, the value of the luminous flux - but also the lifetime depends to a large extent on the stability of the supply voltage. Therefore, correct power system should be a thermally stable current source. Unfortunately, the simple power systems, equipped with passive filters are often used as cheap commercial solutions. In those types of systems stability of the output voltage is heavily dependent on the load conditions. Passive filters - usually consisting of coils and capacitors - are placed in the output circuit. This paper presents research carried out to show of filter capacitance influence on flickering phenomenon, and practical light's flickering measuring technique for the LED lamps.
\end{abstract}

\section{Introduction}

The light emitting diodes (LEDs) is a type of semiconductor diode that emits light when voltage is applied. LED's diode light flux is a function of physical properties of the construction material and the value of the current flowing through the semiconductor. LED chips - commonly used in lamps - consists of two basic blocks: power and optical. Power electronic converter system and control system are part of the power block. While, the optical block can be divided into LED diode, heat sink, lens system, and in some cases a phosphor layer. So, besides temperature, stability of the light flux depends on the power block [1].

\subsection{Light Flickering}

Flickering of lighting is fast, periodic changes in light intensity, so it can become a hazard to health. Flickering caused by inadequate power supply of LEDs is an undesirable

\footnotetext{
* Corresponding author: dsobczyn@prz.edu.pl
} 
phenomenon which has negative impact on human comfort. Besides slow flickering of the LED light sources (components with a frequency not exceeding $80 \mathrm{~Hz}$ ) it is important that emitted light do not contain higher frequency components which are causing the phenomenon of the so called ,spectral matrix". This phenomenon is based on the impression of serial, spatial extension of the point light source after a step change of observed object (leaves impression of the trace of light) [2]. Many LED light sources flickers with frequencies from 100 to $500 \mathrm{HZ}$, which is not noticeable if eye does not move. However during fast eye movements (saccades) images of the LED lights sources appear in different places on the retina which are causing the impression of the image traces.

\subsection{Human Effects of Light Flickering}

The most unfavourable flicker effect occurs in the frequency range from 3 to $70 \mathrm{~Hz}-$ in 1 out of 20000 cases it can lead to epileptic seizures. Although most of the flickering effects occur on the level equal or twice higher than $\mathrm{AC}$ input frequency and is invisible to the human eye, nevertheless this phenomenon can still be a threat. Flickering for the frequencies from the range of 100 to $500 \mathrm{~Hz}$ are causing headache and eyestrain in large part of the population. Invisible flickering is forbidden in certain places such as Hospitals and offices [3]. The cause of invisible flickering are the low harmonic components of the output voltage of the switched power supplies used to power the diodes. Also, there can be other factors as well such as incorrectly working feedback loop, incorrect filter or converter working with current's discontinuity in inductance.

\subsection{LED supply and flickering}

Various types of power supplies and integrated electronic circuits can be used to power supply LED lamps. LED lightning requires constant current - so the key to eliminating light flickering is the right type of the power electronic converter used for power supply. Cheaper, power supplies of lower quality do not provide current stabilization for LED. Instead of providing constant current cheaper versions simply rectify current from AC to DC using a passive filter with LC elements. As a result voltage and current with relatively large ripple - usually with twice the input voltage - will be generated. That generate 100 potential flickers per second.

\section{Mathematical definition of flicker index}

The light flickering is mainly characterized by the depth of luminance changes. The depth of flicker can be quantified using mathematical formulas. There are many differently defined indicators of ripple depth in literature [4,5]:

$$
\begin{gathered}
W_{1}=(\text { Amax }- \text { Bmin }) /(\text { Amax }+B \operatorname{Bmin}) \\
W_{2}=(\text { Amax }- \text { Bmin }) / A m a x \\
W_{3}=(\text { Amax }- \text { Aav }) / A a v \\
W_{4}=(\text { Amax }- \text { Bmin }) / A a v \\
W_{5}=\text { Bmin } / A a v
\end{gathered}
$$




$$
W_{6}=0,5(\text { Amax }-B \min ) / A a v
$$

Where, Amax, Bmin, Aav, marks successively: maximum, minimum and average luminance. The $W_{1}$ index is most often used but expressed as a percentage:

$$
W_{1 \%}=100 \%(\operatorname{Amax}-B \min ) /(\operatorname{Amax}+B \min )
$$

The quantification methods of $W_{1}-W_{6}, W_{1 \%}$ and $F I$ was shown in Fig. 1.

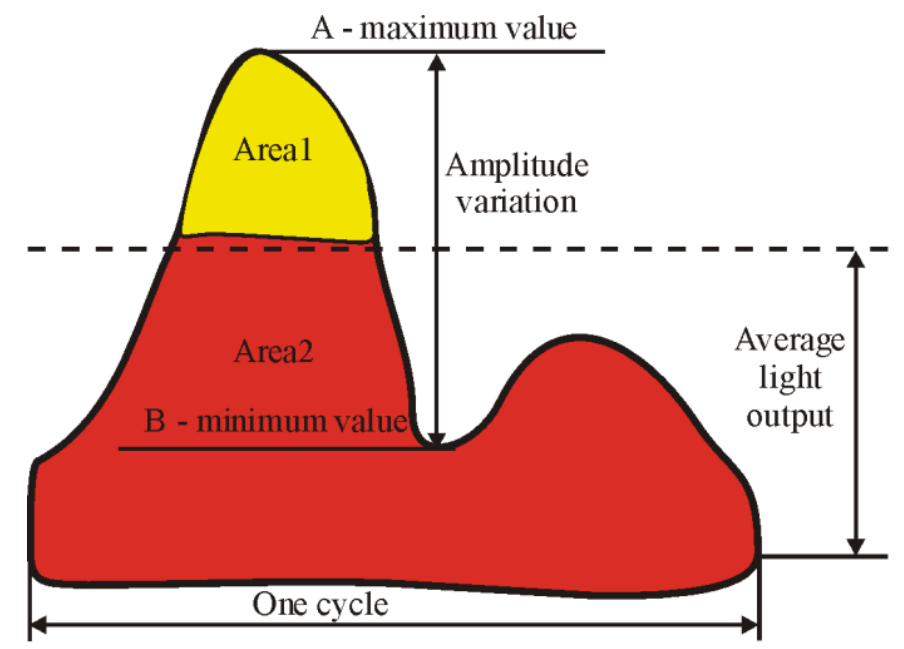

Fig. 1. Flicker quantification methods.

The $W_{1 \%}$ factor does not take into account the shape, fill factor and frequency of the signal. Hence the flicker index $(F I)$ has been used which - in contrast to the percentage of the flicker $W_{1 \%}-$ also takes into account the shape and duty ratio. This coefficient is a better way to describe irregularly shaped courses because of it is able to account for differences in waveform shape [7]:

$$
F I=(\text { Area } 1 /(\text { Area } 1+\text { Area } 2)
$$

The light flicker factors assumes values from 0 to 1 . The value of this coefficient depends on the type of light source used, as well as on its supply system. The flickering of light with the value $W_{1}=0$, can be considered harmless. In practice, this value is greater than zero. It was assumed that the light sources used to illuminate the work areas should have a flicker factor not greater than $W_{1}=0.05$.

In spot lighting, flicker index should be in the range of $0.1<W_{1}<0.15$, while in other illuminated areas this coefficient should be less than 0.2 [7]. As cited before, flicker factor allows for simply and rapid comparison of different LEDs' drivers.

\section{Flicker measurements - experimental setup}

The measuring device uses a digital, light to voltage sensor TSL250R, powered from a lithium-polymer battery. The sensor supply system is additionally equipped with a battery charging module using a micro USB port. The output voltage of the sensor, which is 
directly proportional to changes in light intensity, is given to one of the oscilloscope channels (Fig.2).

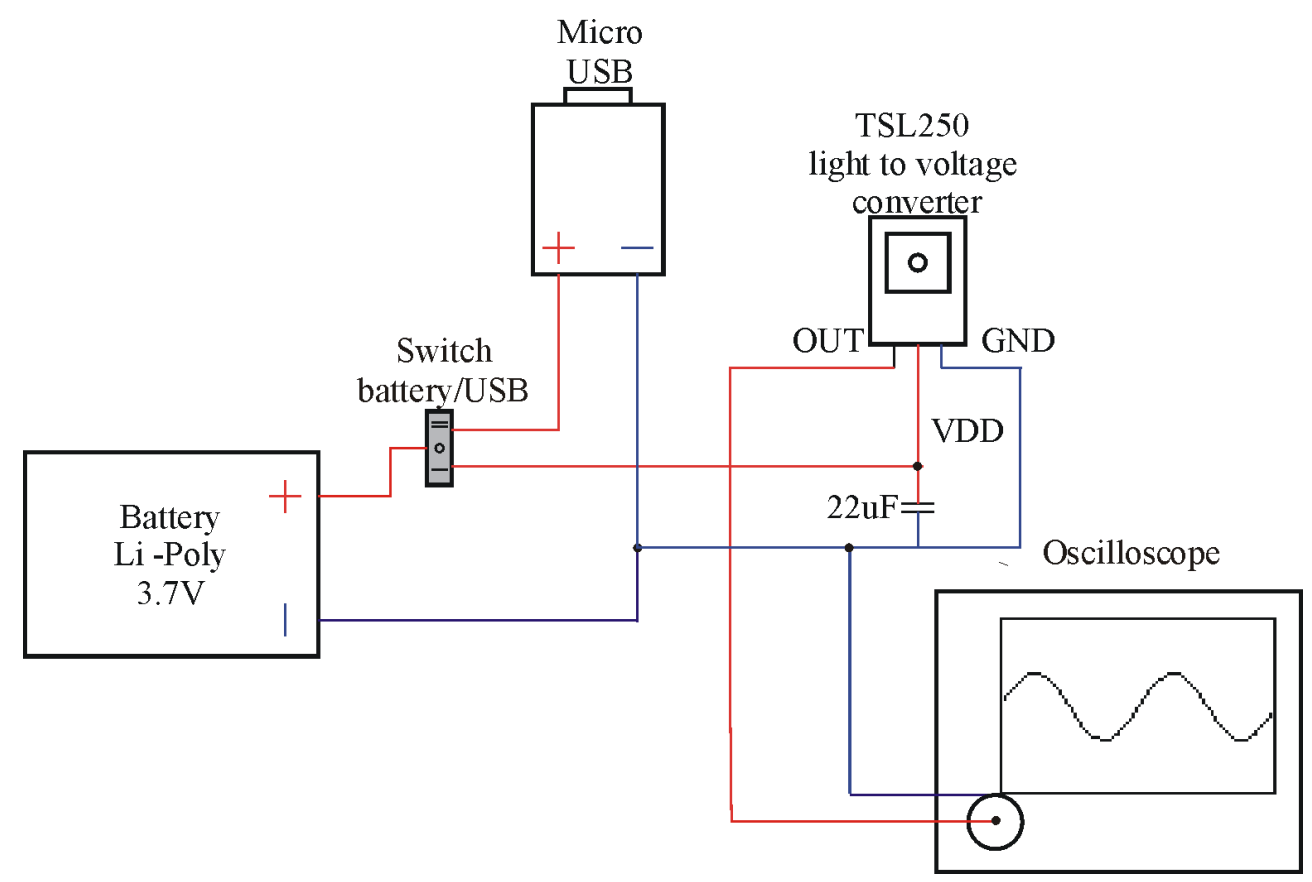

Fig. 2. Measuring circuit.

The LED power converter consists of the following elements (Fig. 3):

- KBPC5010 bridge rectifier.

- Capacitors with capacities of $110 \mu \mathrm{F}, 220 \mu \mathrm{F}$ and $470 \mu \mathrm{F}$.

- 2-position switch.

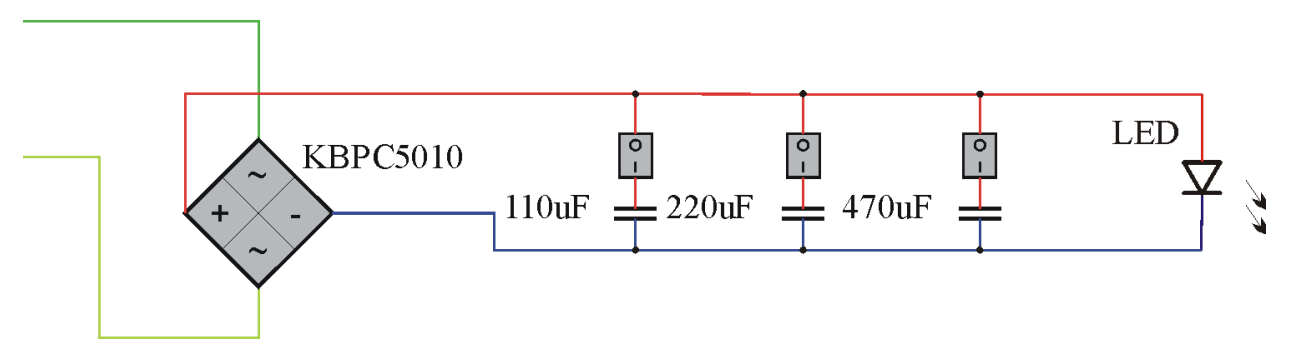

Fig. 3. LED supplier with adjustable capacitor filter.

\section{Laboratory test}

The measurements were carried out in experiment setup presented in Fig 4. The laboratory stand consists of:

- measuring module TSL250,

- amperemeter for measuring LED current,

- voltmeter for measuring the LED supply voltage,

- oscilloscope. 


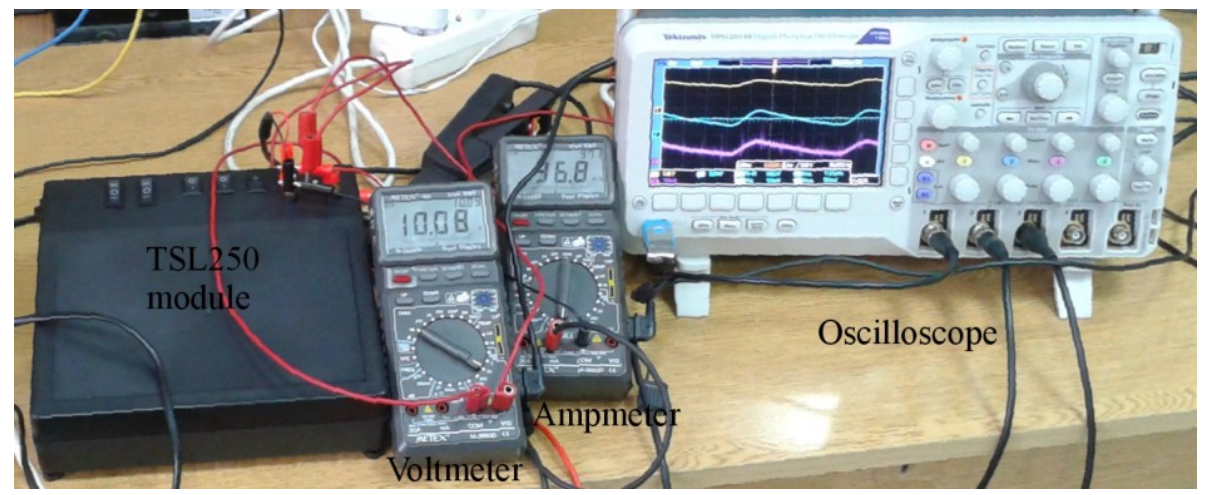

Fig. 4. Experimental setup.

a

c

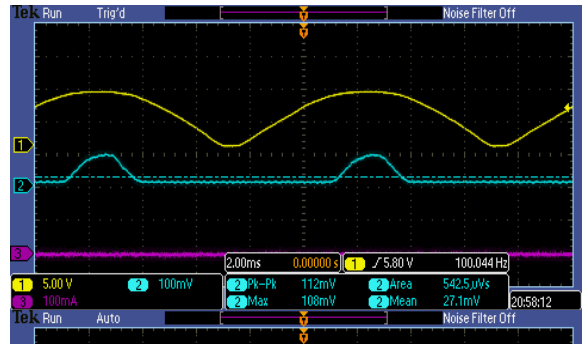

e

g
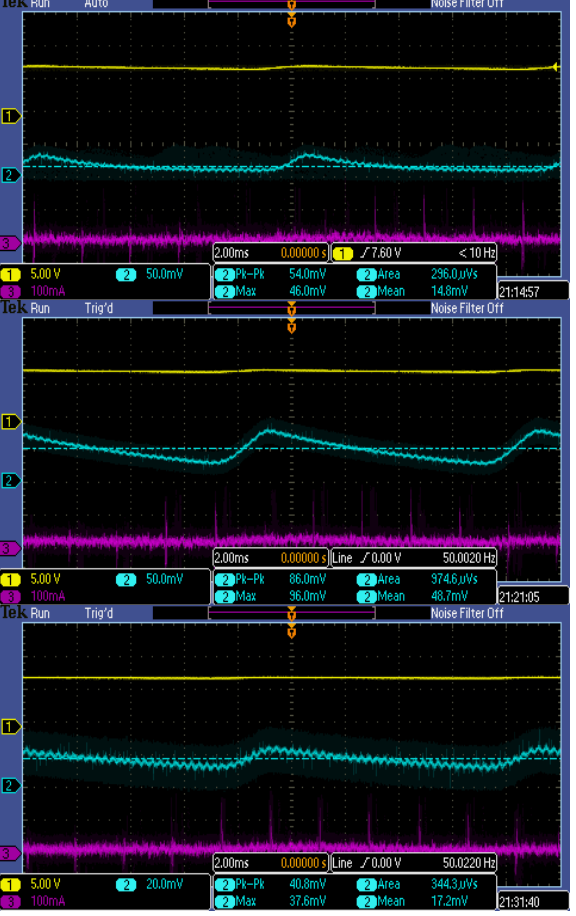

b

d

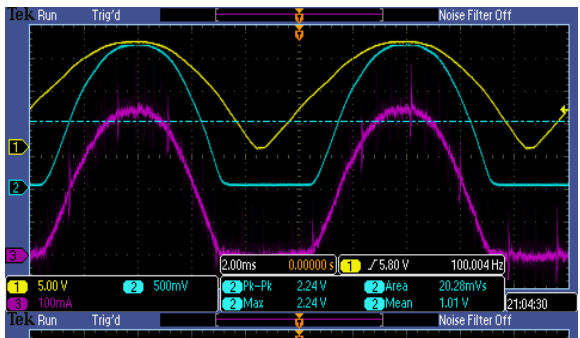

h

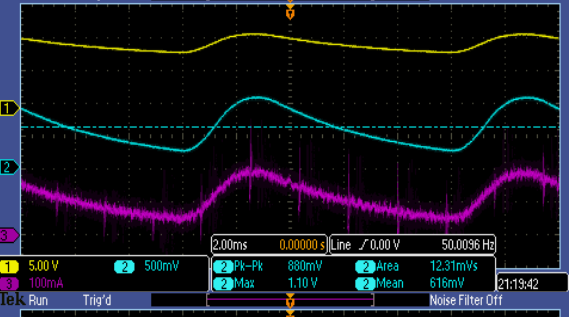

f

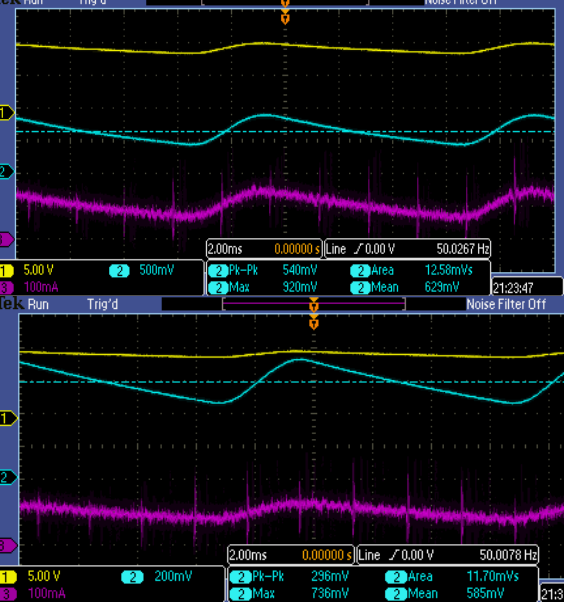

Fig. 5. Waveforms of: diode voltage (Ch1), TSL250 sensor output voltage - proportional to light intensity (Ch2), diode current (Ch3) for different value of supply voltage and filter's capacitor. (a) $\mathrm{U}=$ $7,5 \mathrm{~V} ; \mathrm{C}=0 \mu \mathrm{F}$, (b) $\mathrm{U}=10 \mathrm{~V} ; \mathrm{C}=0 \mu \mathrm{F}$, (c) $U=7,5 \mathrm{~V} ; C=220 \mu \mathrm{F}$, d) $U=10 \mathrm{~V} ; C=220 \mu \mathrm{F}$, (e) $U=7,5 \mathrm{~V}$; $C=470 \mu \mathrm{F}, \mathrm{f}) U=10 \mathrm{~V} ; C=470 \mu \mathrm{F},(\mathrm{g}) U=7,5 \mathrm{~V} ; C=800 \mu \mathrm{F}, \mathrm{h}) U=10 \mathrm{~V} ; C=800 \mu \mathrm{F}$. 
The LEDs' chips and TSL 250 module were mounted in a black box. Each measurement experiments, have been done in order to determination of the light flicker coefficient for different value of passive filter capacitor capacitance. The first aspect that can be analyzed from the tests is a comparison between the all LED flicker indexes for several capacitor filter values. The Fig. 5a to Fig. 5h show qualitatively that some discrepancies in flicker index rise when using various voltage level.

During the measurements, the values of current and voltage supplied to the LED strip were read. On the oscilloscope, the voltage and current waveforms as well as the signal output of the TSL250 transducer proportional to the luminous flux were observed. Measurements have been made during LED strip supplying by converter without an output filter and when filter has been connected subsequently with capacitance vary from $110 \mu \mathrm{F}$ up to $800 \mu \mathrm{F}$. The peak-to-peak, maximum and average values of the light to voltage TSL250 converter output signal have been read. The Area 1 and Area 2 necessary to designate the $F I$ are read from the oscilloscope waveforms (Fig. 5a $-5 \mathrm{~h}$ ), similarly as was shown in Fig. 1.

Table 1. List of values of $W_{1}-W_{6}, W_{1 \%}, F I$ for different values of filter capacitance.

\begin{tabular}{|c|c|c|c|c|c|c|c|c|}
\hline $\begin{array}{c}\text { Filter } \\
\text { capacitance } \\
(\mathrm{uF})\end{array}$ & $W_{1}$ & $W_{2}$ & $W_{3}$ & $W_{4}$ & $W_{5}$ & $W_{6}$ & $W_{1 \%}$ & $F I$ \\
\hline 0 & 1 & 1 & 1.22 & 2.22 & 0 & 1.11 & 100 & 0.402 \\
\hline 110 & 0.94 & 0.97 & 1.16 & 2.1 & 0.06 & 1.05 & 94 & 0.333 \\
\hline 220 & 0.67 & 0.8 & 0.79 & 1.43 & 0.36 & 0.71 & 67 & 0.195 \\
\hline 330 & 0.53 & 0.69 & 0.6 & 1.11 & 0.49 & 0.55 & 53 & 0.141 \\
\hline 470 & 0.42 & 0.59 & 0.46 & 0.86 & 0.6 & 0.43 & 42 & 0.113 \\
\hline 580 & 0.34 & 0.5 & 0.36 & 0.69 & 0.67 & 0.34 & 34 & 0.089 \\
\hline 690 & 0.28 & 0.44 & 0.3 & 0.57 & 0.72 & 0.29 & 28 & 0.075 \\
\hline 800 & 0.25 & 0.4 & 0.26 & 0.51 & 0.75 & 0.25 & 25 & 0.063 \\
\hline
\end{tabular}

The indexes $W_{1}-W_{6}, W_{1 \%}$, provided in Chapter 2 are intended to describe an alternating signal. In the considered case the LED was powered by rectified voltage, which had the variable component, and therefore a description of the phenomenon used those indicators. On the basis of the realised research it can be concluded that the values of $W_{1}-W_{4}, W_{6}$ coefficients, and $W_{1 \%}$, start to decrease with increasing voltage and current supplying the LED, while the $W_{5}$ coefficient starts to increase.

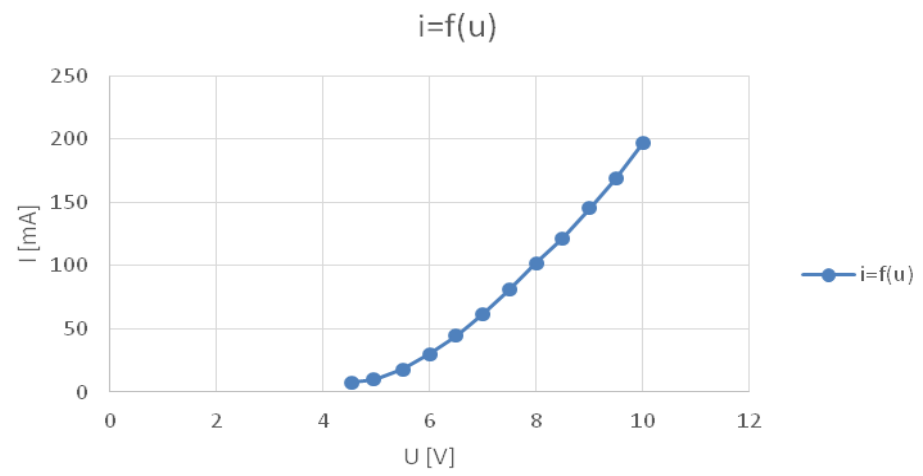

Fig. 6. Diode current-voltage characteristics. 
The flicker indexes have been calculated according to the relationship $(1-8)$ from Chapter 2. Table 1 shows such comparison.

Relating the values of the mentioned factors to the diode current-voltage characteristics, one can also observe a relationship (especially for the $W_{3}-W_{6}$, coefficients) that the changes of those values are getting smaller and smaller from the point where the operating point on the current-voltage characteristics begins to enter linear area (a current of around 30$40 \mathrm{~mA}$ ). Analyzing the $F I$ index can be notice that its value (similar to the $W_{1}-W_{4}$, and $W_{6}$, coefficients) decreases with the increase of current and LED voltage, however these changes in relation to other coefficients are so small that one can accept that this ratio is constant for a given capacity. The factors values are decreasing together with the increase of voltage and supply current. That may confirm necessity of proper voltage supply parameter sets which were given by the producers.

The calculated indexes from $W_{1}-W_{6}$, have a value greater than 1 . After attaching subsequent capacities, the values of the indices are within the range given in the literature, from 0 to 1 . Below, the values of flicker at measuring points at voltage of $10 \mathrm{~V}$ are presented, where the values are the smallest for individual capacities and they are presented in Fig. 7.

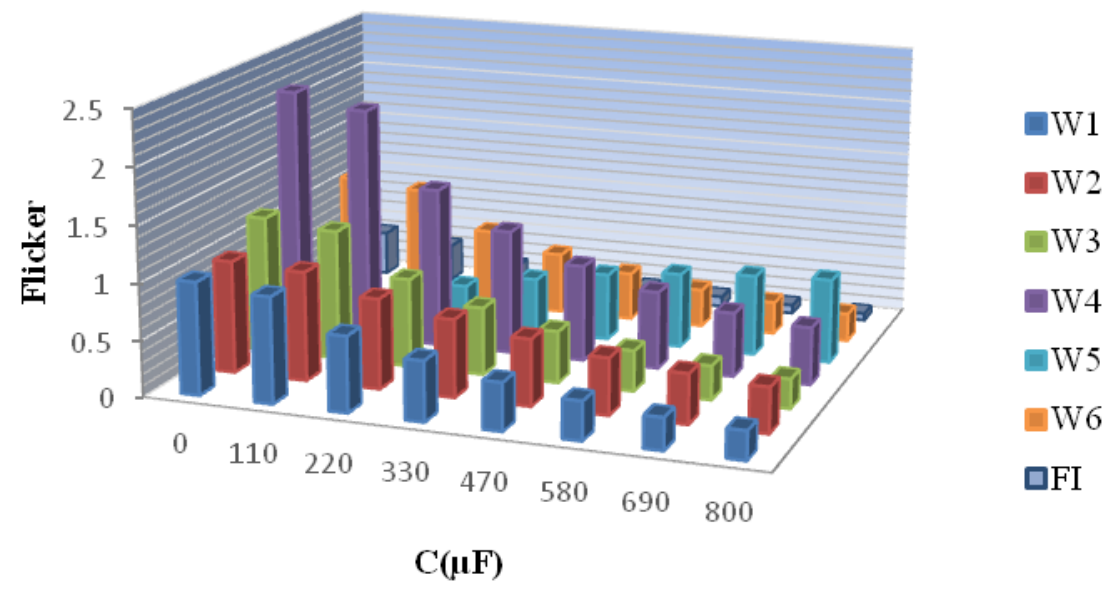

Fig. 7. List of flicker indexes for different capacitances.

As can be seen in the case under consideration, the flicker light indexes have reach the lowest values for the largest volume of capacitor, equal to $800 \mu \mathrm{F}$.

\section{Conclusion}

After analyzing obtained flicker index values it has been concluded that increasing the output capacity of LED power supply filter (reducing voltage ripples) results in reducing the ripple of light. The flicker index values from $W_{1}$ to $W_{6}$ and $W_{1 \%}$ do not fit into acceptable limits specified in the Chapter 2. Assuming that values of the flicker index $F I$, are also regulated by quoted limited values, so can be conclude that given LED can be used as a light source with the filter capacity of $220 \mu \mathrm{F}(F I<0.2)$. Using diode lamp in local lightning would require to attach a minimum capacity of $330 \mu \mathrm{F}(F I<0.15)$. For the capacity value of $800 \mu \mathrm{F}$ flicker index $F I$ equals 0.063 which exceeds acceptable value of 0.05 used to illuminate exact workplaces. In reference to maximum flicker indexes provided by IEEE, given value meets the condition with the capacity of $690 \mu \mathrm{F}$ or higher $(F I<08)$. As mentioned in Section 2, FI takes into account the shape and duty ratio of the signal which 
contrast with the other factors that as pointed in literature on the subject are used to describe the ripples of light caused by AC power supply. Obtained values of the factors $W_{1}$ $-W_{6}, W_{1 \%}, F I$ differ from the values given in Chapter 2 . Test results confirm the validity of utilizing FI factor - especially for the rectified voltage shape. It can be noticed that this coefficient was within the limits defined in the theoretical part of this article, differently than other factors.

\section{References}

1. X. Cao, D. Zheng, 11 th China Int. For. on Solid State Lighting, Inteligent LED lighting system and sensor technology, (2014)

2. W.A. Hershberger, J.S. Jordan, The Psych. Rec., The Phantom array: a perisaccadic illusion of visual direction, 48, 21-32 (1998)

3. A. J. Wilkins, J. Veitch, B. Lehman, IEEE standard PAR1789 update, in Proc. IEEE Energy Conversion Congr. Expo., LED lighting flicker and potential health concerns, 171-178 (2010)

4. R. Roy, Richtek Application Note AN022, Minimizing Light Flicker in LED Lighting Applications, (2014)

5. M. Poplawski, N.J. Miller, Pacific Northwest National Laboratory Tech. Rep., (2013)

6. J. Gomez, M. Morcos, IEEE Power Engineering Review, Flicker measurement and light effect, 11-15, (2002).

7. A. Wiśniewski, Przegląd Elektrotechniczny, Tętnienie światła wytwarzanego przez Tuby LED - zamienniki świetlówek liniowych, 7 (2015) 